\title{
Immune and Stress Responses Covary with Melanin-Based Coloration in the Barn Swallow
}

\author{
Nicola Saino • Luca Canova • Alessandra Costanzo • \\ Diego Rubolini • Alexandre Roulin • \\ Anders Pape Møller
}

Received: 5 February 2013/ Accepted: 22 March 2013/Published online: 17 April 2013

(C) Springer Science+Business Media New York 2013

\begin{abstract}
Eumelanin and pheomelanin are the main endogenous pigments in animals and melanin-based coloration has multiple functions. Melanization is associated with major life-history traits, including immune and stress response, possibly because of pleiotropic effects of genes that control melanogenesis. The net effects on pheo- versus eumelanization and other life-history traits may depend on the antagonistic effects of the genes that trigger the biosynthesis of either melanin form. Covariation between melanin-based pigmentation and fitness traits enforced by pleiotropic genes has major evolutionary implications particularly for socio-sexual communication. However, evidence from non-model organisms in the wild is limited to very few species. Here, we tested the hypothesis that melanin-based coloration of barn swallow (Hirundo rustica) throat and belly feathers covaries with acquired immunity and activation of the hypothalamic-pituitaryadrenal (HPA) axis, as gauged by corticosterone plasma levels. Individuals of both sexes with darker brownish belly
\end{abstract}

N. Saino $(\bowtie) \cdot$ A. Costanzo $\cdot$ D. Rubolini

Department of Biosciences, University of Milan, via Celoria 26, 20133 Milan, Italy

e-mail: nicola.saino@unimi.it

L. Canova

Department of Biology and Biotechnology "Lazzaro Spallanzani", Università degli Studi di Pavia, via Ferrata 9, 27100 Pavia, Italy

\section{A. Roulin}

Department of Ecology and Evolution, Biophore, University of Lausanne, 1024 Lausanne, Switzerland

\section{A. P. Møller}

Laboratoire Ecologie, Systematique et Evolution, Batiment 362, 91405 Orsay Cedex, France feathers had weaker humoral immune response, while darker males had higher circulating corticosterone levels only when parental workload was experimentally reduced. Because color of belly feathers depends on both eu- and pheomelanin, and its darkness decreases with an increase in the concentration of eu- relative to pheomelanin, these results are consistent with our expectation that relatively more eu- than pheomelanized individuals have better immune response and smaller activation of the HPA-axis. Covariation of immune and stress response arose for belly but not throat feather color, suggesting that any function of color as a signal of individual quality or of alternative lifehistory strategies depends on plumage region.

\section{Keywords Corticosterone - Color - Hirundo rustica .} Immunity $\cdot$ Melanin $\cdot$ Sex

\section{Introduction}

Melanins are the main endogenous pigmentary determinants of coloration in animals (Majerus 1998). Melanization of the integument serves a widely diverse set of functions, from protection of body tissues from abrasion and solar radiation to camouflage of both prey and predators and communication (Roulin 2004; Hill and McGraw 2006; Hoekstra 2006; see Meunier et al. 2011). The dissection of the natural selection forces that have molded the evolution of melanism in individual species/populations is therefore deemed to be complex.

A fact of potentially major importance in the evolution of melanism is that the genes ultimately underpinning the regulation of melanin biosynthesis pleiotropically influence several life-history traits via the melanocortin system (Ducrest et al. 2008). Melanocortins are derivatives of the prohormone 
encoded by the proopiomelanocortin $(P O M C)$ gene. Binding of the POMC gene products to the melanocortin 1-receptor (MC1R), which is expressed in the melanocytes of hair and feather follicles as well as in several tissues, triggers eumelanogenesis. In vertebrates, however, melanin pigmentation most often results from the accumulation of the black eumelanin and the yellow-reddish brown alternative melanin form, pheomelanin. The relative allocation to production of either component of such 'mixed melanin' pigmentation is mainly regulated by binding of melanocortins and the agouti signaling protein (ASIP) to MC1R, whereby ASIP inhibits production of eumelanin and triggers pheomelanin synthesis (see Ducrest et al. 2008; Simon et al. 2009).

Pleiotropic effects of POMC arise because its peptide derivatives, as well as ASIP can also bind to other MC (MC25) receptors besides $\mathrm{MC} 1 \mathrm{R}$, exerting regulatory effects on a number of additional important functions, spanning from energy homeostasis and socio-sexual behavior to immunity and stress response via the hypothalamic-pituitary-adrenal (HPA) axis (Ducrest et al. 2008). ASIP, however, has antagonistic effects to melanocortins, implying that opposite correlations should arise between such functions and euversus pheomelanogenesis (Roulin et al. 2011). Several studies have shown that by binding to MC1R and also MC3R and MC5R melanocortins can influence the course of inflammatory and cytotoxic immune processes in model species. Moreover, the fact that MC1R is expressed in several immune cell types (Catania et al. 1996; Taherzadeh et al. 1999; Loser et al. 2010; see Gangoso et al. 2011) suggests that melanocortins can have an important role in orchestrating both cell-mediated and humoral immunity.

Few studies on immune function and coloration in the wild have been carried out, and most of them understandably refer to immature individuals before fledging (Ducrest et al. 2008). In the tawny owl (Strix aluco), when nestlings were subjected to a humoral immune challenge the intensity of a subsequent $\mathrm{T}$ cell-mediated cutaneous response to phytohemagglutinin (PHA), but not the intensity of an innate immune response to bacterial LPS, was enhanced in nestlings with dark plumage and the opposite was the case for pale nestlings (Gasparini et al. 2009). Moreover, a difference in the norm of reaction to PHA among nestlings was recorded according to maternal pheomelanic pigmentation. In contrast, Eleonora's falcon (Falco eleonorae) male nestlings of the dark morph mounted a weaker immune response to PHA than females (Gangoso et al. 2011). In the barn owl (Tyto alba), offspring with more spotted eumelanic plumage were found to express a larger humoral immune response to an experimental immune challenge (Roulin et al. 2000). Darker adult female tawny owls mount a more persistent antibody response to vaccination (Gasparini et al. 2009). Moreover, darker tawny owls experience lower parasitaemia (Galeotti and Sacchi 2003) and show different reaction norms to food stress (Piault et al. 2009).
Melanocortins are also involved in regulation of the stress response via ACTH, which is part of the HPA axis that activates the synthesis of glucocorticoids by binding to MC2R in the adrenal glands (Charmandari et al. 2005; Ducrest et al. 2008). Evidence for covariation between melanization and stress response via the HPA also comes from studies of very few species in the wild (Ducrest et al. 2008). For example, among barn owl nestlings, the pattern of daily corticosterone ('Cort') level variation depended on eumelanic coloration of their parents (Roulin et al. 2010) and post-handling stress Cort levels were lower among more eumelanic individuals, suggesting better ability to cope with stress (Almasi et al. 2010). Moreover, administration of Cort caused higher growth rate of more eumelanic nestlings (Almasi et al. 2012). However, baseline circulating Cort level was invariant with pheo- and eumelanization of adult male barn owls, although more eumelanic males had a smaller Cort-induced reduction in parental performance (Almasi et al. 2008).

Thus, pleiotropy of $P O M C$ and antagonistic effects of ASIP may provide a mechanistic basis for the associations between melanism and life-history traits (Ducrest et al. 2008). Yet, with the exception of a couple of closely related species where the potential associations among melanism and life-history traits has been studied, there is little information from vertebrate populations in the wild. This gap of knowledge is particularly pronounced for adult individuals. However, melanogenesis and coloration are known to vary with age (Weimerskirch et al. 1989; Potti and Montalvo 1991; Galván and Møller 2009). Immune functions are also known to vary during ontogeny (Janeway et al. 2005). In addition, physiological coping styles to stress can change during an individual's life (Rensel et al. 2010).

The association between melanism and major fitness traits is of great relevance to evolutionary studies and particularly to the investigation of communication systems mediated by signals that reveal individual genetic quality and/or variation in life strategies with similar adaptive value (Majerus 1998; Jawor and Breitwisch 2003; Roulin 2004; Roulin et al. 2011). Therefore, there is a need to assess the generality of the facts that have been documented in some species.

In this study we take advantage of two previous experimental studies of a passerine bird, the barn swallow (Hirundo rustica), to investigate covariation between immune function or activation of the HPA axis (Saino et al. 2002a, 2003), and melanin-based coloration. As markers of immune function we used antibody response to an antigenic challenge and a general indicator of $\mathrm{T}$ cell-mediated immune response (the 'PHA test'). As a marker of the activation of the HPA axis, we used pre-acute stress (hereafter 'baseline') Cort levels at the end of the nestling period measured on a sample 
of fathers whose brood was enlarged or, respectively, reduced to alter parental workload (Saino et al. 2002a). Furthermore, we applied a restraint stress protocol to trigger a short-term elevation in Cort levels (see Wingfield et al. 1998; Almasi et al. 2010; Liebl and Martin 2012) and investigated covariation between coloration and the increase in Cort levels following restraint.

Coloration was quantified by spectrophotometry for two plumage regions: the reddish-brown throat patch and the whitish-to-brownish belly plumage. These feathers contain both pheo- and eumelanin in barn swallows of the nominate $H$. rustica from Scotland (throat feathers only; McGraw et al. 2004) and of the subspecies H. r. erythrogaster from North America (belly feathers only; McGraw et al. 2005). A recent study of barn swallows from the same population where the present study has been conducted (Saino et al. 2013) has confirmed that feathers from both plumage regions contain both melanin forms. Coloration is predicted by the concentration of both melanins in belly feathers of both sexes. Coloration of throat feathers, however, is predicted only by the concentration of pheomelanin in females, with larger concentrations being associated with darker, more saturated brown coloration. Importantly, the proportion of pheo- to eumelanin concentration positively predicts darkness and saturation of belly feather color (Saino et al. 2013), suggesting that coloration reflects allocation to pheo- relative to eumelanogenesis.

Because brown coloration depends on both pheo- and eumelanin absolute concentrations, and melanocortin and their ASIP antagonist are expected to exert opposite effects, we had no clear-cut expectations on the sign of the associations between color components and immune or stress response. However, color darkness and saturation of whitish to brown belly feathers is positively predicted by the ratio between pheo- and eumelanin concentrations, implying that darker, more saturated color reflects larger allocation to pheo- than to eumelanogenesis (see above). Thus, we could expect weaker immune response in individuals with darker, relatively more pheo- than eumelaninc belly or throat (females) feathers (Ducrest et al. 2008).

Because baseline Cort levels are involved in the resolution of trade-offs between major fitness traits, including reproduction (Sapolsky et al. 2000; Romero 2004; Charmandari et al. 2005; Landys et al. 2006; Bonier et al. 2009; Roulin et al. 2010) and are also involved in the regulation of circadian activity rhythms (Pancak and Taylor 1983; Jessop et al. 2002), we expected the consequences of experimentally altered brood size to differ depending on melanization. Although the consequences of chronic stress on Cort levels may be difficult to predict, we expected higher Cort levels in darker males because their color reflects larger allocation to pheo- than eumelanogenesis, and particularly so among males attending enlarged broods. Similarly, we expected positive covariation between darkness and Cort levels after restraint stress, with a steeper relationship among males attending enlarged broods.

These predictions on the association between darkness of 'visible' feather coloration and immune or stress response variables pertain to the $\theta$ hue tetrahedral color component (see "Methods"). Because in barn swallow belly feathers $\theta$ tends to be negatively correlated with the $\varphi$ color component, accounting for UV reflectance, and with chroma, the predictions for $\varphi$ and chroma were formulated by adjusting their sign for this pattern of association among color components.

\section{Methods}

We relied on immune response and Cort plasma concentration data recorded during two experiments carried out in 1999 and 2001. The results of these studies are reported in Saino et al. (2002a, 2003). No information on coloration was reported in the previously published papers.

\section{Primary Antibody Response to NDV Vaccination}

The methodological details of vaccination and antibody response analyses are reported in details in Saino et al. (2003). Briefly, in 2001 we captured, sexed and individually marked breeding adults at six breeding colonies (=farms) located east of Milano (Northern Italy). Each individual received a subcutaneous injection of $20 \mu \mathrm{l}$ commercial NDV vaccine (Nobivac ${ }^{\circledR}$ Paramyxo, Intervet, Salamanca, Spain). Vaccination occurred prior to egg laying; then individuals were captured 2 weeks after vaccination and a blood sample was collected to measure humoral response to vaccination. NDVspecific antibody concentration was measured by monoclonal antibody-blocking enzyme-linked immunosorbent assay (Czifra et al. 1996; Saino et al. 2002b, 2003). The intensity of the immune response was expressed as percentage inhibition values, which are proportional to NDV-specific antibody concentrations. Age of breeding adults was identified based on well-established procedures (see e.g. Saino et al. 2003, 2012 and references therein).

\section{Wing Web Swelling Response to Phytohemagglutinin}

The full details of the experiment that we did in 1999 where we measured the cutaneous response to PHA and Cort plasma levels are reported in Saino et al. (2002a). The experiment aimed at analyzing the effect of altered parental effort on T cell-mediated immune response and Cort levels both before and after an acute stress episode (i.e. restraint in a bag) (Saino et al. 2002a). Post-acute stress Cort levels 
are presented here for the first time. We manipulated the size of the first brood of individually marked males immediately after hatching by reciprocally swapping an unbalanced number of nestlings between pairs of synchronous broods. We thus produced a group of broods whose size was reduced by one nestling and a group of broods whose size was increased by one nestling, while also establishing a control group of unmanipulated broods (PHA test only). Males were captured between 04:40 and 07:00 a.m., on average 1.9 days after fledging (6.3 SD; range 7 days before-16 days after fledging) of their nestlings in May to mid-June. After blood sampling for Cort analyses (see below), they were subjected to inoculation of $0.2 \mathrm{mg}$ of PHA dissolved in $0.04 \mathrm{ml}$ of phosphate-buffered saline (PBS) in the right wing web while the same amount of PBS was injected in the left wing web after the thickness of both wing webs had been measured using a pressuresensitive micrometer. The next morning, we recaptured the males and measured again the thickness of both wing webs. The intensity of the immune response was expressed as the difference between the change in thickness of the right, PHA-injected wing web, minus the change recorded in the left, sham-injected wing web (Saino et al. 1997).

\section{Blood Sampling and Corticosterone Analyses}

During the brood size manipulation experiment in 1999, upon the first capture (i.e. the capture when we injected PHA) we took a blood sample $(100 \mu \mathrm{l})$ in capillary tubes by puncturing the ulnar vein. By standing close to the mist nets at the exits of the stables where barn swallows breed and normally spend the night during the breeding period, and by operating in teams of 2-3 people, we managed to sample blood within $1 \mathrm{~min}$ after the bird was captured in the net. Immediately after blood sampling, the birds were put individually in a cloth bag in a safe position at ambient temperature. Forty minutes later, a second blood sample was taken. This protocol was applied to induce an acute stress response. A similar protocol has been adopted by several previous studies (e.g. Almasi et al. (2010) who restrained barn owl nestlings for $26 \pm 6$ (SD) min; Liebl and Martin (2012) who restrained the birds for $30 \mathrm{~min}$ ). Cort concentrations were measured by radioimmunoassay (see Saino et al. 2002a for the details). Males were captured between 04:40 and 07:00 a.m. on average 4.2 days after fledging (6.4 SD; range 7 days before-16 days after fledging) of their nestlings in May to mid-June. Hence, any seasonal, time-of-day or breeding stage effects on circulating corticosterone levels (Breuner et al. 1999; Romero 2002) should have been minimal (see also Saino et al. 2002a). Because offspring are attended by their parents for ca. 1 week after fledging, most males were still involved or had just completed their first-brood parental duties. It should be noted that in the present study we present the data on Cort levels at second blood sampling (40-41 min after capture) that had not been included in Saino et al. (2002a). In the analyses we used 'baseline' Cort levels recorded upon capture and change in Cort levels from baseline to those following restraint for $40 \mathrm{~min}$.

\section{Feather Coloration Measurements}

We collected 3-10 feathers from the medial region of the brown throat color patch and 3-5 feathers from the left paramedian belly plumage region where coloration varies considerably among individuals from white to brownish. The feathers were stored in plastic bags in a dark place at room temperature until color analysis. Reflectance of one belly feather or three overlain throat feathers was measured twice using an Avantes DH-2000 spectrometer in a dark chamber. Illumination was provided by a combined deuterium-tungsten halogen light source. The illuminated field was $2.5 \mathrm{~mm}^{2}$ wide and was centered approximately $1.5 \mathrm{~mm}$ from the distal end of the throat feathers and $2.5 \mathrm{~mm}$ from the distal end of the belly feather (i.e. in a white-to-brownish region). Color was quantified using the tetrachromatic color space model using TetraColorSpace program (Version 1a) run in MATLAB 7 (MathWorks, Natick, MA) (Goldsmith 1990), which incorporates information on both plumage reflectance spectra and bird cone sensitivity. This allows to estimate the relative stimulation of the retinal cones and thus to better model the color perceived by birds. This model has recently been adopted in a number of studies of tetrachromatic vertebrates (e.g. Stoddard and Prum 2008). Each color vector in the tetrahedral color space is transformed to Cartesian coordinates that are subsequently converted into the spherical coordinates $\theta, \varphi$, and $\mathrm{r}$ (see Stoddard and Prum 2008). $\theta$ and $\varphi$ that we used here to quantify hue roughly represent the red-green-blue $(\theta)$ or the ultraviolet $(\varphi)$ components of hue, while $r$ is a measure of chroma or color saturation, which was expressed as 'achieved chroma', $r A=r / r_{\max }$, i.e. as the ratio between measured chroma and maximum potential chroma $\left(r_{\max }\right)$.

In the analyses we assumed UVS cone type-retina and used spectral sensitivity of the blue tit (Cyanistes caeruleus) because this is the species phylogenetically more close to the barn swallow for which spectral sensitivity information is implemented in TetraColorSpace program. However, correlation analysis of $\theta$ and $\varphi$ color components (see below) obtained by assuming blue tit or, respectively, average bird UVS spectral sensitivities disclosed strong associations (details not shown).

Relatively low values of $\theta$ indicate darker brownish hues. Because the $\varphi$ component mainly reflects coloration in the UV spectrum, no verbal description of variation in 
color according to the $\varphi$ component as would be perceived by humans can be provided. Achieved chroma reflects saturation of the color, i.e. its level of 'monochromaticity'.

Repeatability of the coloration variables as estimated by measuring twice the same feather(s) of both plumage regions was always larger than $0.73(n=45$ individuals for both plumage regions). Among-feathers repeatability estimated by measuring two different feathers from the same region was larger than 0.74 for all variables $(n=10$ individuals for both plumage regions).

\section{Statistical Analyses}

We relied on linear models to analyze the association between immune or stress response variables and sex (binary factor) and age and tetrahedral components of feathers coloration (covariates). Because $\theta, \varphi$ and rA color components are inherently correlated within plumage regions while their correlation between plumage regions is low (Saino et al. 2013), we ran separate models where we included only $\theta, \varphi$ or $\mathrm{rA}$ values measured on both plumage regions to avoid incurring biases in parameter estimates due to collinearity. In multiple linear models, when no significant interaction effects were found, interaction terms were removed en bloc in order to reduce the risk of incurring type I statistical errors. In the analyses of antibody response to vaccination in relation to coloration, data were centered within sex to control for any sex-related variation in coloration and immune response. In Cort analyses, the concentrations of the hormone were $\log _{\mathrm{e}}-$ transformed. Throughout the study, the estimated parameters are presented with their standard error in parentheses. The $\alpha$-value of all statistical tests was set at 0.05 . All analyses were run using SPSS 13.0 statistical package.

\section{Results}

Primary Immune Response (PI) to NDV Vaccination and Coloration

In spring 2001 we measured primary immune response to NDV vaccination in 48 males and 28 females. In linear models of PI in relation to sex, age, and color components of belly and throat feathers, antibody response positively covaried with the $\theta$ color component of belly feathers, but not with the other color components of belly feathers or with color of throat feathers (Table 1; Fig. 1). The twoway interaction effects did not attain significance in any case and were therefore removed from the models (Table 1). Specifically, this implies that the slope of the relationships between antibody response and the $\theta$ hue component of belly feather color did not differ between males and females. Because low $\theta$ values are associated with darker brown hues, the positive sign of the coefficient associated with $\theta$ [97.09 (29.82)] indicates that individuals with paler (i.e. less brownish) belly feathers raised a stronger immune response. Anti-NDV antibody concentration declined with age independently of sex and coloration, as indicated by the negative sign of the coefficients that significantly differed from 0 in the models of two out of three color components $[\theta:-5.12(2.27)$; rA: -6.67 (2.35)] (Table 1; see Saino et al. 2003).

Cell-mediated Immune Response to PHA and Coloration

In the experiment (spring 1999) where we manipulated brood size to measure the consequences of parental workload for $\mathrm{T}$ cell-mediated immune response and Cort levels of male parents, we found no significant covariation between PHAresponse and any of the color components measured on feathers from either plumage region (Table 2). Moreover,

Table 1 Primary immune response to NDV immunization in relation to sex (factor), age, and belly or throat feather tetrahedral color components (covariates; see "Methods")

\begin{tabular}{|c|c|c|c|c|c|c|c|}
\hline \multirow{2}{*}{$\begin{array}{l}\text { Color } \\
\text { component }\end{array}$} & \multirow[b]{2}{*}{$d f$} & \multicolumn{2}{|l|}{$\theta$} & \multicolumn{2}{|l|}{$\varphi$} & \multicolumn{2}{|l|}{$\mathrm{rA}$} \\
\hline & & $F$ & $P$ & $F$ & $P$ & $F$ & $P$ \\
\hline Sex & 1.71 & 0.15 & 0.696 & 0.11 & 0.745 & 0.24 & 0.629 \\
\hline Age & 1.71 & 5.11 & 0.027 & 2.89 & 0.094 & 8.03 & 0.006 \\
\hline $\begin{array}{l}\text { Belly color } \\
\text { component }\end{array}$ & 1.71 & 10.60 & 0.002 & 2.31 & 0.133 & 2.54 & 0.115 \\
\hline $\begin{array}{l}\text { Throat color } \\
\text { component }\end{array}$ & 1.71 & 0.27 & 0.603 & 0.85 & 0.360 & 0.05 & 0.825 \\
\hline Sex $\times$ age & 1.65 & 1.74 & 0.192 & 1.76 & 0.190 & 1.97 & 0.165 \\
\hline $\begin{array}{l}\text { Sex } \times \text { belly } \\
\text { color } \\
\text { component }\end{array}$ & 1.65 & 0.39 & 0.536 & 0.00 & 0.966 & 1.10 & 0.297 \\
\hline $\begin{array}{l}\text { Sex } \times \text { throat } \\
\text { color } \\
\text { component }\end{array}$ & 1.65 & 0.23 & 0.635 & 0.00 & 0.958 & 0.30 & 0.587 \\
\hline $\begin{array}{l}\text { Age } \times \text { belly } \\
\text { color } \\
\text { component }\end{array}$ & 1.65 & 0.00 & 0.99 & 0.50 & 0.484 & 0.05 & 0.831 \\
\hline $\begin{array}{l}\text { Age } \times \text { throat } \\
\text { color } \\
\text { component }\end{array}$ & 1.65 & 2.44 & 0.123 & 0.32 & 0.573 & 0.37 & 0.546 \\
\hline $\begin{array}{l}\text { Belly } \times \text { throat } \\
\text { color } \\
\text { components }\end{array}$ & 1.65 & 0.07 & 0.789 & 0.82 & 0.368 & 0.83 & 0.366 \\
\hline
\end{tabular}

For all color variables, statistics for two-way interaction terms were obtained from multiple linear models including main effects. Statistics for main effects were obtained from multiple linear models from which all interaction terms had been removed, as their effect was nonsignificant (see "Statistical analyses"). Sample sizes: $\mathrm{n}=28$ males; $\mathrm{n}=48$ females. See "Results" for parameter estimates of the significant terms, which are bolded 


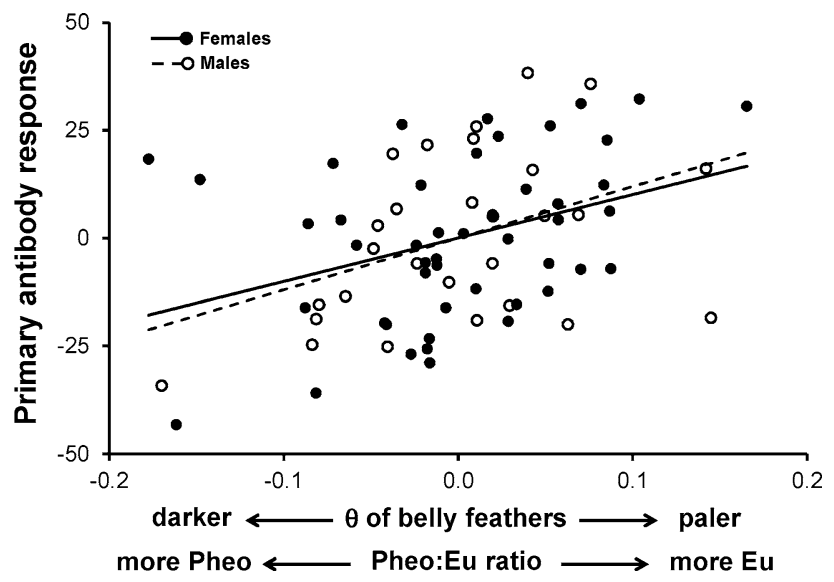

Fig. 1 Primary immune response to NDV vaccine (expressed as percentage inhibition) in relation to the $\theta$ color component of belly feathers in male and female barn swallows. The direction of change in darkness of brownish visible color and of the relative pheo- and eumelanin concentrations (according to Saino et al. 2013) are displayed on the $x$-axis. Lines are fitted by linear regression. The relationship is significant for both sexes $(P<0.05$; see "Results")

PHA response was not predicted by the interaction effects between brood size manipulation and color (Table 2). Information on age was available for 30 out of the 34 males that were involved in the analyses. When the main effects of age and interaction terms with color components were included in the models presented in Table 2, no significant effects emerged (details not shown).

\section{Corticosterone Plasma Levels and Coloration}

In the same experiment (spring 1999) where we measured PHA-response after brood size manipulation, baseline Cort concentrations of parental males were found to covary in a complex way with color. Cort levels were predicted by the interaction between brood size manipulation and $\theta$ of belly feathers (Table 3). A simplified model including only brood size manipulation and $\theta$ of belly feathers confirmed a significant interaction $\left(F_{1,39}=7.48, P=0.009\right.$; see also Table 3 ) and showed that Cort concentrations declined as $\theta$ increased (i.e. declined as belly feathers got paler) in males attending reduced broods [coefficient: -3.42 (1.39), $t=$ $-2.47, \quad P=0.018]$, whereas they non-significantly increased with $\theta$ [coefficient: $0.94(0.97), t=0.98, P=0.335$ ] in males attending an enlarged brood (Fig. 2). Cort levels were also predicted by the interaction between brood size manipulation and $\mathrm{rA}$, reflecting color saturation (Table 3). Cort levels increased with saturation in both experimental groups, but more steeply so among males with a reduced compared to an enlarged brood [coefficient for brood reduction: 29.25 (9.13), $t=3.20, P=0.003$; enlargement: 23.33 (9.15), $P=0.015$ ]. In the same model, there was also a significant interaction effect between rA measured in belly or throat feathers. The coefficient associated with the interaction between rA terms $[-77.94$ (27.46)] indicates that Cort levels declined more steeply for relatively large values of $\mathrm{rA}$ components of belly and throat feathers color.

To test for the effect of exposure to an acute stress episode resulting from restraining the birds in a bag, Cort was also measured on blood samples collected $40 \mathrm{~min}$ after the first sample had been taken immediately after capture. Cort significantly increased [on average by 13.4 (3.73) $\mathrm{ng} \mathrm{m}{ }^{-1}$ ] between the first and the second sample $\left(\log _{\mathrm{e}^{-}}\right.$ transformed data: $t=3.70, d f=42, P=0.001$ ) and this change did not differ between males attending a reduced compared to an enlarged brood $(t=0.67, d f=41$, $P=0.506)$. The relationship between change in Cort and $\theta$ of belly feathers depended on experimental treatment, as indicated by the significant interaction (Table 3). The coefficients estimated by the model in Table 3 indicate a non-significant negative covariation in reduced broods [coefficient: -4.101 (4.312), $t=-0.95, P=0.348$ ] and a significant negative covariation for enlarged broods [-8.900 (4.089), $t=-2.18, P=0.036]$, indicating higher

Table 2 Wing web swelling response to PHA injection (reflecting T cell-mediated immune response) in relation to brood size manipulation (three-levels factor: reduced, control, enlarged) and color components of belly or throat feathers (see "Methods")

\begin{tabular}{|c|c|c|c|c|c|c|c|}
\hline \multirow[t]{2}{*}{ Color component } & \multirow[b]{2}{*}{$d f$} & \multicolumn{2}{|l|}{$\theta$} & \multicolumn{2}{|l|}{$\varphi$} & \multicolumn{2}{|l|}{$\mathrm{rA}$} \\
\hline & & $F$ & $P$ & $F$ & $P$ & $F$ & $P$ \\
\hline Brood size manipulation (BSM) & 2.29 & 3.09 & 0.061 & 3.84 & 0.033 & 3.15 & 0.058 \\
\hline Belly color component & 1.29 & 0.26 & 0.614 & 0.82 & 0.374 & 0.16 & 0.691 \\
\hline Throat color component & 1.29 & 0.19 & 0.668 & 0.05 & 0.824 & 0.00 & 0.949 \\
\hline $\mathrm{BSM} \times$ belly color component & 2.24 & 0.25 & 0.781 & 0.10 & 0.909 & 0.88 & 0.427 \\
\hline $\mathrm{BSM} \times$ throat color component & 2.24 & 0.14 & 0.871 & 0.07 & 0.934 & 0.284 & 0.755 \\
\hline Belly $\times$ throat color components & 2.24 & 0.41 & 0.526 & 0.078 & 0.782 & 0.01 & 0.908 \\
\hline
\end{tabular}

For all color variables, statistics for two-way interaction terms were obtained from multiple linear models including main effects. Statistics for main effects were obtained from multiple linear models from which all interaction terms had been removed, as their effect was non-significant (see "Statistical analyses"). Sample size of males were: brood reduction: $\mathrm{n}=14$, unmanipulated: $\mathrm{n}=11$, brood enlargement: $\mathrm{n}=9$. As information on age was available for 30 out of the 34 individuals, models excluding the non-significant age effects are presented (see "Results") 
Table 3 Baseline and post-acute stress change in corticosterone levels at the end of the nestling period in relation to brood size manipulation (two-levels factor: reduced, enlarged) and color components of belly or throat feathers

\begin{tabular}{|c|c|c|c|c|c|c|c|c|c|}
\hline \multirow[t]{2}{*}{ Color component } & \multicolumn{3}{|l|}{$\theta$} & \multicolumn{3}{|l|}{$\varphi$} & \multicolumn{3}{|l|}{$\mathrm{rA}$} \\
\hline & $d f$ & $F$ & $P$ & $d f$ & $F$ & $P$ & $d f$ & $F$ & $P$ \\
\hline \multicolumn{10}{|l|}{ Baseline } \\
\hline Brood size manipulation (BSM) & 1.36 & 0.65 & 0.427 & 1.39 & 0.64 & 0.427 & 1.36 & 0.37 & 0.549 \\
\hline Belly color component & 1.36 & 0.15 & 0.705 & 1.39 & 0.00 & 0.959 & 1.36 & 8.45 & 0.006 \\
\hline Throat color component & 1.36 & 0.00 & 0.953 & 1.39 & 2.72 & 0.107 & 1.36 & 10.14 & 0.003 \\
\hline $\mathrm{BSM} \times$ belly color component & 1.36 & 5.95 & 0.020 & 1.36 & 0.01 & 0.925 & 1.36 & 4.87 & $\mathbf{0 . 0 3 4}$ \\
\hline $\mathrm{BSM} \times$ throat color component & 1.36 & 0.04 & 0.852 & 1.36 & 1.16 & 0.289 & 1.36 & 0.23 & 0.636 \\
\hline Belly $\times$ Throat color components & 1.36 & 0.82 & 0.371 & 1.36 & 2.46 & 0.125 & 1.36 & 8.06 & 0.007 \\
\hline \multicolumn{10}{|l|}{ Change after restraint } \\
\hline Brood size manipulation (BSM) & 1.36 & 1.21 & 0.278 & 1.39 & 0.51 & 0.481 & 1.36 & 0.09 & 0.767 \\
\hline Belly color component & 1.36 & 2.59 & 0.116 & 1.39 & 0.16 & 0.696 & 1.36 & 9.68 & 0.004 \\
\hline Throat color component & 1.36 & 3.56 & 0.067 & 1.39 & 0.03 & 0.859 & 1.36 & 8.93 & 0.005 \\
\hline $\mathrm{BSM} \times$ belly color component & 1.36 & 4.32 & 0.045 & 1.36 & 0.15 & 0.700 & 1.36 & 3.76 & 0.060 \\
\hline $\mathrm{BSM} \times$ throat color component & 1.36 & 0.00 & 0.957 & 1.36 & 1.60 & 0.215 & 1.36 & 0.12 & 0.729 \\
\hline Belly $\times$ throat color components & 1.36 & 3.86 & 0.057 & 1.36 & 2.26 & 0.142 & 1.36 & 9.73 & 0.004 \\
\hline
\end{tabular}

For the $\varphi$ color component, statistics for two-way interaction terms were obtained from multiple linear models including main effects, while statistics for main effects were obtained from multiple linear models from which all interaction terms had been removed, as their effect was nonsignificant (see "Statistical analyses"). For $\theta$ and rA, all the effects were estimated from multiple linear models including all terms, as some interaction effects were significant. Sample size of males were: brood reduction: $n=24$, enlargement: $n=19$. See "Results" for parameter estimates of the significant terms, which are bolded

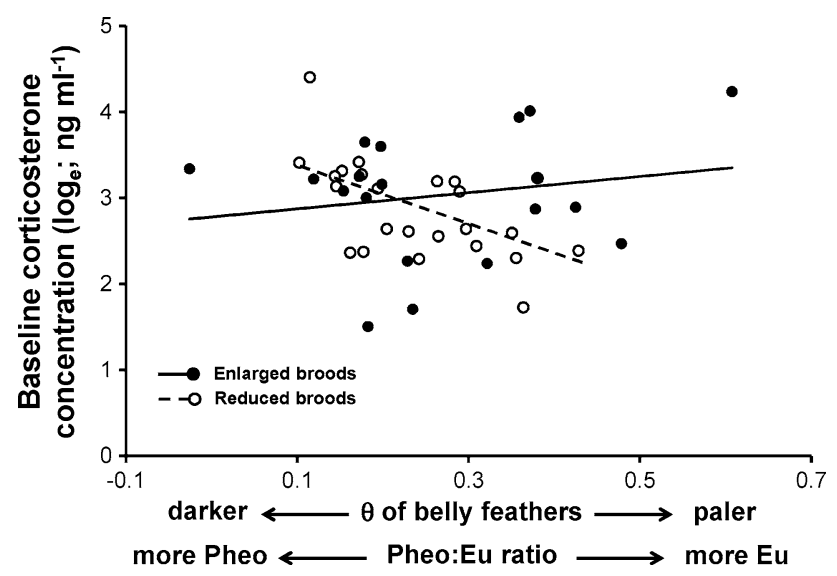

Fig. 2 'Baseline' corticosterone plasma levels recorded immediately after capture in relation to the $\theta$ color component of belly feathers of male barn swallows that were attending a reduced or an enlarged brood. The direction of change in darkness of brownish visible color and of the relative pheo- and eumelanin concentrations (according to Saino et al. 2013) are displayed on the $x$-axis. Lines are fitted by linear regression. The relationship for reduced broods is statistically significant $(P<0.05$; see "Results")

stress-induced Cort levels in darker individuals. This discrepancy between the positive trend in the raw data for reduced broods (Fig. 3) and the coefficient estimated by the model (Table 3) likely depends on the non-negligible, though statistically non-significant effect of other terms in the model. Finally, there was a strong interaction effect between $\mathrm{rA}$ values recorded at either plumage region on change in Cort levels. The positive coefficient associated with the interaction term [117.793 (37.765)] indicates that change in Cort increased disproportionately for high rA values of both throat and belly feathers.

\section{Discussion}

Pleiotropic effects of the genes that control melanogenesis have been invoked as the ultimate cause of the covariation between major life-history traits and melanic coloration (Ducrest et al. 2008). However, there is limited information on covariation between immune function or HPA-mediated stress response and melanism in adults from free-ranging organisms (see Introduction). We aimed at filling this gap of knowledge by analyzing data on humoral and $\mathrm{T}$ cellmediated immune response and on stress response mediated by the HPA axis in relation to plumage coloration in adult barn swallows. We found that antibody response to a novel antigen was less intense in individuals of both sexes with relatively dark brownish belly plumage. This relationship was independent of any age effects. In addition, we can exclude seasonal or habitat effects (see Gasparini et al. 2009) because color and immunity did not vary with date nor among colonies in the present sample (details not shown). 


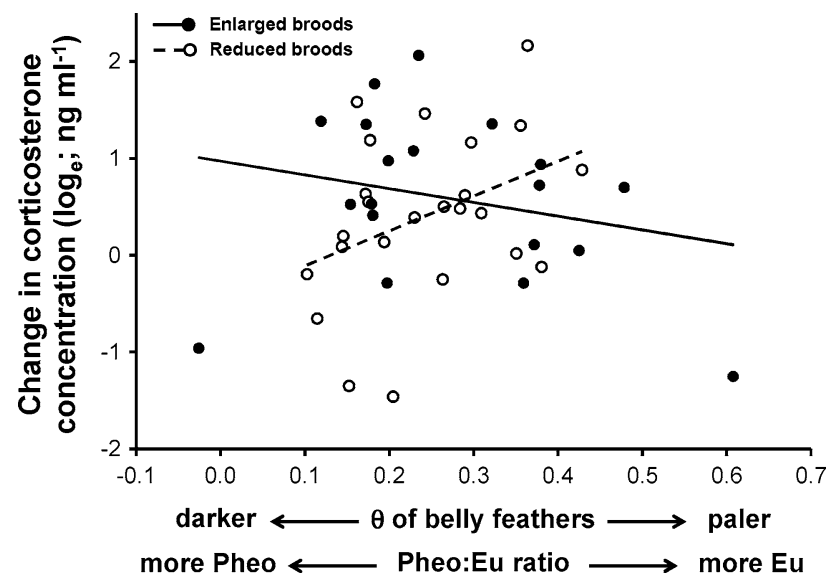

Fig. 3 Change in corticosterone plasma levels between the time of capture and after 40 min restraint in relation to the $\theta$ tetrahedral color component of belly feathers of male barn swallows that were attending a reduced or an enlarged brood. The direction of change in darkness of brownish visible color and of the relative pheo- and eumelanin concentrations (Saino et al. 2013) are displayed on the $x$-axis. Lines are fitted by linear regression. The relationship for enlarged broods is statistically significant $(P<0.05$; see "Results")

In the barn swallow, as in other species with mixed melanic pigmentation like the tawny owl (Gasparini et al. 2009), darkness of coloration of individual brownish feathers depends on the absolute concentration of eu- and pheomelanin, making it difficult to formulate clear-cut predictions on the sign of the covariation between performance and color. This is the case because the pleiotropic genetic mechanisms that underpin eu- and pheomelanogenesis may have reciprocally antagonistic effects on performance traits (Ducrest et al. 2008; Roulin et al. 2011). However, darkness is significantly positively predicted by the relative concentration of pheo- and eumelanin in males (Saino et al. 2013), while this relationship is also positive but non-significant among females. This implies that, in our study, individuals with larger allocation to eu- relative to pheomelanogenesis had larger humoral immune response. Because the genes that trigger eu- or pheomelanogenesis have reciprocally antagonistic effects, the net effect on the traits that they are pleiotropically targeting may depend on the relative levels of their simultaneous expression. Because paler individuals are relatively more eumelanic (Saino et al. 2013), the present evidence of more intense immune response by paler individuals is consistent with the expectation of better immune performance associated with eumelanization. In general, it could be suggested that relative, besides absolute levels of eu- or pheomelanization should be considered in the analysis of the covariation between lifehistory traits and melanization. Although in species with mixed melanization relative and absolute levels of eu- (or pheo-) melanins will inevitably tend to be positively correlated, the relative concentration of either melanin form, besides their absolute concentrations, may also predict performance at other, genetically linked traits. Because of the mixed (eu- plus pheomelanin) nature of the melanization of barn swallow feathers, the mechanistic interpretation of the covariation between humoral immunity and color hue is not straightforward. Yet, the present results are consistent with a general model of pleiotropic, antagonistic effects of the genes that trigger eu- and pheomelanogenesis, and also with a positive association between eumelanogenesis and immunity because individuals with larger concentration of eumelanin relative to pheomelanin had more intense humoral response.

Response to PHA in barn swallows declines after fledging, but variance among adults is extensive (coefficient of variation $=86 \%$; present study), implying that there is ample scope for a covariation with other traits. However, we did not find any association of response to PHA with coloration, which was expected because PHA-response is associated with mutations at MC1R (Gangoso et al. 2011). We have no firm explanation for the lack of association between melanization and PHA response. In general, pleiotropic effects of genes that control melanization are expected to result in behavioral and physiological syndromes. Therefore, the resolution of physiological tradeoffs between the competing demands of immune response and socio-sexual activities or reproduction (Bonneaud et al. 2003; Uller et al. 2006) could itself covary with melanization. Such tradeoff could thus have confounded any association between melanization and this component of acquired immunity.

Because of pleiotropy of the genes that ultimately control melanogenesis, we also analyzed variation in Cort levels after altered parental workload or acute stress, according to color. Parents attending size-manipulated broods adjust their food provisioning effort (Saino et al. 2000). In the present study, workload of parents of enlarged broods was ca. $20 \%$ larger than that of parents of reduced broods (Saino et al. 2002a). This modest increase in parental effort was not associated with any apparent effect on baseline Cort levels. Yet, we found increased activation of the HPA axis in darker adult males attending a reduced, but not an enlarged brood. The present results for male parents of reduced broods can be interpreted along the same line as those for antibody response (see above): because darkness depends on concentration of both pheomelanin and eumelanin, but increases with the pheo- to eumelanin ratio, our results indicate that males that allocate more to eu- than to pheomelanogenesis have lower activation of the HPA axis. Why no relation was observed among parental males of enlarged broods is matter of speculation. In fact, predicting the consequences of longlasting altered stimulation of the HPA axis-mediated coping mechanism for Cort levels is difficult. One possibility is that the resolution of tradeoffs among e.g. parental care and 
socio-sexual behavior under increased parental effort covaried with coloration. This is consistent with previously reported interaction effects between brood size manipulation and color on offspring growth (Roulin et al. 2008a, b). In addition, covariation between melanin coloration and other phenotypic traits are very often context-dependent (Roulin 2009).

Stressful restraint (see Almasi et al. 2010; Liebl and Martin 2012) caused an increase in Cort levels, consistent with the prediction. However, change in Cort levels did not differ between brood size manipulation treatments. This finding indicates that altered parental workload does not influence subsequent susceptibility to a novel stressor possibly because the level of stress was too mild. Alternatively, the effect of acclimation to chronic stress on one hand and any positive feedback on HPA axis responsiveness during increased parental workload on the other cancelled each other, producing no differential effect on postrestraint Cort levels between treatments. Post-restraint, however, relatively more pheomelanic fathers of enlarged broods had larger Cort levels, again consistently with the prediction that individuals that allocate more to eu- than pheomelanogenesis should show lower activation of the HPA axis.

Evidence for higher resistance to stressors in dark eumelanic individuals has been found in studies of vertebrate species in the wild (Ducrest et al. 2008). For example, studies of barn owls have suggested that coping styles of darker eumelanic individuals allow better buffering of the physiological consequences of stress for activation of the HPA axis (Roulin and Ducrest 2011; but see Almasi et al. 2008). Hence, as long as relative eu- and pheomelanin levels are considered, present results are consistent with the expectation, although the specific patterns of covariation between Cort and color depending on experimental brood size are puzzling.

Unexpectedly, while significant covariation of immune and stress responses was observed for the 'visible' hue of belly feathers, there was no association with hue of throat feathers. This finding may suggest that melanogenesis of different plumage regions is regulated by partly different mechanisms. Feathers are organized in feather tracts that are molted at different times of the annual cycle (Ginn and Melville 1983). Feather tracts also commonly differ in coloration. In addition, these color patterns show specific sex- and age-related changes (e.g. Galván and Møller 2009). Different feather tracts with colors based on different pigments often constitute multiple signals with different information content (Møller and Pomiankowski 1993). Experiments on chicken cell cultures have shown that melanosomes migrate from the underlying tissue to the feather follicles depending on the antioxidant status of the tissue (Bowers et al. 1999). Therefore, different feather tracts such as the throat and the belly feathers of the barn swallow may differ in their susceptibility to stress and, therefore, also in their relationship with immune function.

As long as any potential environmental determination of melanogenesis is considered (Fargallo et al. 2007), temporal effects might also concur in this differential covariation because belly and head feathers are molted at partly different times (Turner 2006). However, it is difficult to imagine how such asynchrony in molt could result in a difference in the association between coloration at either plumage region and fitness traits, given that molt is completed months before the breeding season.

The difference in the association between melanization at different plumage regions and physiological traits has obvious implications for the analysis of the socio-sexual function of coloration, as it indicates that belly but not throat coloration may reflect genetic quality or individual differences in life-history strategies with similar adaptive value (Roulin 2004). On the other hand, Cort levels covaried with the interaction effects between chroma of belly and throat feathers, suggesting that signaling may also occur via the combined color saturation at either plumage region.

In conclusion, we provided evidence for an association between melanin-based coloration, humoral immunity and activation of the HPA axis in response to chronic and acute stress. These findings are consistent with a general model of pleiotropic effects of genes that control melanogenesis. Indeed, we observed that darker individuals, which accumulate relatively more pheo- than eumelanin in their belly feathers compared to paler ones, had weaker humoral immune response and higher activation of the HPA axis, although the latter association depended on experimental manipulation of parental workload. Significant covariation between immune or stress response variables and color was observed for belly, but not throat, feathers. Therefore, these findings suggest that any socio-sexual signaling function of individual quality, as well as any differences in life-history strategies with similar adaptive values, mediated by melanization, vary among plumage regions.

\section{References}

Almasi, B., Jenni, L., Jenni-Eiermann, S., \& Roulin, A. (2010). Regulation of stress response is heritable and functionally linked to melanin-based coloration. Journal of Evolutionary Biology, 23, 987-996.

Almasi, B., Roulin, A., Jenni-Eiermann, S., \& Jenni, L. (2008). Parental investment and its sensitivity to corticosterone is linked to melanin-based coloration in barn owls. Hormones and Behaviour, 54, 217-223.

Almasi, B., Roulin, A., Korner-Nievergelt, F., Jenni-Eiermann, S., \& Jenni, L. (2012). Coloration signals the ability to cope with 
elevated stress hormones: Effects of corticosterone on growth of barn owls is associated with melanism. Journal of Evolutionary Biology, 25, 1189-1199.

Bonier, F., Martin, P. R., Moore, I. T., \& Wingfield, J. C. (2009). Do baseline glucocorticoids predict fitness? Trends in Ecology \& Evolution, 24, 634-642.

Bonneaud, C., Mazuc, J., Gonzalez, G., Haussy, C., Chastel, O., Faivre, B., et al. (2003). Assessing the cost of mounting an immune response. American Naturalist, 161, 367-379.

Bowers, R. R., Nguyen, B., Buckner, S., Gonzalez, Y., \& Ruiz, F. (1999). Role of antioxidants in the survival of normal and vitiliginous avian melanocytes. Cellular and Molecular Biology, $45,1065-1074$.

Breuner, C. W., Wingfield, J. C., \& Romero, L. M. (1999). Diel rhythms of basal and stress-induced corticosterone in a wild, seasonal vertebrate, Gambel's white-crowned sparrow. Journal of Experimental Zoology, 284, 334-342.

Catania, A., Rajora, N., Capsoni, F., Minonzio, F., Star, R. A., \& Lipton, J. M. (1996). The neuropeptide a-MSH has specific receptors on neutrophils and reduces chemotaxis in vitro. Peptides, 17, 675-679.

Charmandari, E., Tsigos, C., \& Chrousos, G. (2005). Endocrinology of the stress response. Annual Review of Physiology, 67, 259-284.

Czifra, G., Nilsson, M., Alexander, D. J., Manvell, R., Kecskeméti, S., \& Engström, B. E. (1996). Detection of PMV-1 specific antibodies with a monoclonal antibody blocking enzyme-linked immunosorbent assay. Avian Pathology, 25, 691-703.

Ducrest, A.-L., Keller, L., \& Roulin, A. (2008). Pleiotropy and the melanocortin system, coloration and behavioural syndromes. Trends in Ecology \& Evolution, 23, 502-510.

Fargallo, J. A., Laaksonen, T., Korpimäki, E., \& Wakamatsu, K. (2007). A melanin-based trait reflects environmental growth conditions of nestling male Eurasian kestrels. Evolutionary Ecology, 21, 157-171.

Galeotti, P., \& Sacchi, R. (2003). Differential parasitaemia in the tawny owl (Strix aluco): Effects of colour morph and habitat. Journal of Zoology, 261, 91-99.

Galván, I., \& Møller, A. P. (2009). Different roles of natural and sexual selection on senescence of plumage colour in the barn swallow. Functional Ecology, 23, 302-309.

Gangoso, L., Grande, J. M., Ducrest, A.-L., Figuerola, J., Bortolotti, G. R., Andrés, J. A., et al. (2011). MC1R-dependent, melaninbased colour polymorphism is associated with cell-mediated response in the Eleonora's falcon. Journal of Evolutionary Biology, 24, 2055-2063.

Gasparini, J., Piault, R., Bize, P., \& Roulin, A. (2009). Synergistic and antagonistic interaction between different branches of the immune system is related to melanin-based coloration in nestling tawny owls. Journal of Evolutionary Biology, 22, 2348-2353.

Ginn, H. B., \& Melville, D. S. (1983). Moult in birds. Tring: British Trust for Ornithology.

Goldsmith, T. H. (1990). Optimization, constraint, and history in the evolution of eyes. Quarterly Review of Biology, 65, 281-322.

Hill, G. E., \& McGraw, K. J. (2006). Bird coloration. Volume II. Function and evolution. Cambridge: Harvard University Press.

Hoekstra, H. E. (2006). Genetics, development and evolution of adaptive pigmentation in vertebrates. Heredity, 97, 222-234.

Janeway, C. A., Travers, P., Walport, M., \& Shlomchik, M. J. (2005). Immunobiology: The immune system in health and disease (6th ed.). New York: Garland Science Publishing.

Jawor, J. M., \& Breitwisch, R. (2003). Melanin ornaments, honesty, and sexual selection. Auk, 120, 249-265.

Jessop, T. S., Limpus, C. J., \& Whittier, J. M. (2002). Nocturnal activity in the green sea turtle alters daily profiles of melatonin and corticosterone. Hormones and Behaviour, 41, 357-365.
Landys, M. M., Ramenofsky, M., \& Wingfield, J. C. (2006). Actions of glucocorticoids at a seasonal baseline as compared to stressrelated levels in the regulation of periodic life processes. General and Comparative Endocrinology, 148, 132-149.

Liebl, A. L., \& Martin, L. B. (2012). Exploratory behaviour and stress hyper-responsiveness facilitate range expansion of an introduced bird. Proceedings of the Royal Society of London B-Biological Sciences, 279, 4375-4381.

Loser, K., Brzoska, T., Oji, V., Auriemma, M., Voskort, M., Kupas, V., et al. (2010). The neuropeptide alpha-melanocyte-stimulating hormone is critically involved in the development of cytotoxic CD8+T cells in mice and humans. PLoS ONE, 5, e8958.

Majerus, M. E. N. (1998). Melanism, evolution in action. Oxford: Oxford University Press.

McGraw, K. J., Safran, R. J., Evans, M. R., \& Wakamatsu, K. (2004). European barn swallows use melanin pigments to color their feathers brown. Behavioral Ecology, 5, 889-891.

McGraw, K. J., Safran, R. J., \& Wakamatsu, K. (2005). How feather colour reflects its melanin content. Functional Ecology, 19, 816-821.

Meunier, J., Figueuredo Pinto, S., Burri, R., \& Roulin, A. (2011). Eumelanin-based coloration and fitness parameters in birds: A meta-analysis. Behavioral Ecology and Sociobiology, 65, $559-567$.

Møller, A. P., \& Pomiankowski, A. (1993). Why have birds got multiple sexual ornaments? Behavioral Ecology and Sociobiology, 32, 167-176.

Pancak, M. K., \& Taylor, D. H. (1983). Seasonal and daily plasma corticosterone rhythms in American toads, Bufo americanus. General and Comparative Endocrinology, 50, 490-497.

Piault, R., Gasparini, J., Bize, P., Jenni-Eiermann, S., \& Roulin, A. (2009). Pheomelanin-based coloration and the ability to cope with variation in food supply and parasitism. American Naturalist, $174,548-556$.

Potti, J., \& Montalvo, S. (1991). Male color variation in Spanish pied flycatchers Ficedula hypoleuca. Ibis, 133, 293-299.

Rensel, M. A., Broughton, R. K., \& Schoech, S. J. (2010). Development of the adrenal stress response in the Florida scrub jay (Aphelocoma coerulescens). General and Comparative Endocrinology, 165, 255-261.

Romero, L. M. (2002). Seasonal changes in plasma glucocorticoid concentrations in free-living vertebrates. General and Comparative Endocrinology, 128, 1-24.

Romero, L. M. (2004). Physiological stress in ecology: Lessons from biomedical research. Trends in Ecology \& Evolution, 19, 249-255.

Roulin, A. (2004). The evolution, maintenance and adaptive function of genetic colour polymorphism in birds. Biological Reviews, 79, $815-848$.

Roulin, A. (2009). Covariation between eumelanic pigmentation and body mass only under specific conditions. Naturwissenschaften, 96, 375-382.

Roulin, A., Almasi, B., \& Jenni, L. (2010). Temporal variation in glucocorticoid levels during the resting phase is associated in opposite way with maternal and paternal melanic coloration. Journal of Evolutionary Biology, 23, 2046-2053.

Roulin, A., Almasi, B., Meichtry-Stier, K. S., \& Jenni, L. (2011). Eumelanin- and pheomelanin-based colour advertise resistance to oxidative stress in opposite ways. Journal of Evolutionary Biology, 24, 2241-2247.

Roulin, A., Almasi, B., Rossi-Pedruzzi, A., Ducrest, A.-L., Wakamatsu, K., Miksik, I., et al. (2008a). Corticosterone mediates the condition-dependent component of melanin-based coloration. Animal Behaviour, 75, 1351-1358.

Roulin, A., \& Ducrest, A.-L. (2011). Association between melanism, physiology and behaviour: A role for the melanocortin system. European Journal of Pharmacology, 660, 226-233. 
Roulin, A., Gasparini, J., Bize, P., Ritschard, M., \& Richner, H. (2008b). Melanin-based colorations signal strategies to cope with poor and rich environments. Behavioral Ecology and Sociobiology, 62, 507-519.

Roulin, A., Jungi, T. W., Pfister, H., \& Dijkstra, C. (2000). Female barn owls (Tyto alba) advertise good genes. Proceedings of the Royal Society of London B-Biological Sciences, 267, 937-941.

Saino, N., Calza, S., \& Møller, A. P. (1997). Immunocompetence of nestling barn swallows (Hirundo rustica) in relation to brood size and parental effort. Journal of Animal Ecology, 66, 827-836.

Saino, N., Ferrari, R. P., Martinelli, R., Romano, M., Rubolini, D., \& Møller, A. P. (2002b). Early maternal effects mediated by immunity depend on sexual ornamentation of the male partner. Proceedings of the Royal Society of London B-Biological Sciences, 269, 1005-1009.

Saino, N., Ferrari, R. P., Romano, M., Rubolini, D., \& Møller, A. P. (2003). Humoral immune response in relation to senescence, sex and sexual ornamentation in the barn swallow (Hirundo rustica). Journal of Evolutionary Biology, 16, 1127-1134.

Saino, N., Incagli, M., Martinelli, R., \& Møller, A. P. (2002a). Immune response of male barn swallows in relation to parental effort, corticosterone plasma levels, and sexual ornamentation. Behavioral Ecology, 13, 169-174.

Saino, N., Ninni, P., Incagli, M., Calza, S., Sacchi, R., \& Møller, A. P. (2000). Begging and parental care in relation to offspring need and condition in the barn swallow (Hirundo rustica). American Naturalist, 156, 637-649.

Saino, N., Romano, M., Ambrosini, R., Rubolini, D., Boncoraglio, G., Caprioli, M., et al. (2012). Longevity and lifetime reproductive success of barn swallow offspring are predicted by their hatching date and phenotypic quality. Journal of Animal Ecology, 81, 1004-1012.
Saino, N., Romano, M., Rubolini, D., Teplitski, C., Ambrosini, R., Caprioli, M., et al. (2013). Sexual dimorphism in melanin pigmentation, feather coloration and its heritability in the barn swallow (Hirundo rustica). PLoS ONE, 8, e58024.

Sapolsky, R. M., Romero, L. M., \& Munck, A. U. (2000). How do glucocorticoids influence stress responses? Integrating permissive, suppressive, stimulatory, and preparative actions. Endocrinological Reviews, 21, 55-89.

Simon, J. D., Peles, D., Wakamatsu, K., \& Ito, S. (2009). Current challenges in understanding melanogenesis: Bridging chemistry, biological control, morphology, and function. Pigment Cell \& Melanoma Research, 22, 563-579.

Stoddard, M. S., \& Prum, R. O. (2008). Evolution of avian plumage color in a tetrahedral color space: A phylogenetic analysis of new world buntings. American Naturalist, 171, 755-776.

Taherzadeh, S., Sharma, S., Chhajlani, V., Gantz, I., Rajora, N., Demitri, M. T., et al. (1999). a-MSH and its receptors in regulation of tumor necrosis factor-a production by human monocyte/macrophages. American Journal of Physiology-Regulatory Integrative and Comparative Physiology, 276, R1289-R1294.

Turner, A. (2006). The barn swallow. London: T. \& A. D Poyser.

Uller, T., Isaksson, C., \& Olsson, M. (2006). Immune challenge reduces reproductive output and growth in a lizard. Functional Ecology, 20, 873-879.

Weimerskirch, H., Lequette, B., \& Jouventin, P. (1989). Development and maturation of plumage in the wandering albatross Diomedea exulans. Journal of Zoology, 219, 411-421.

Wingfield, J. C., Hunt, K., Breuner, C., Dunlap, K., Fowler, G. S., Freed, L., et al. (1998). Environmental stress, field endocrinology, and conservation biology. In J. R. Clemmons \& R. Buchholz (Eds.), Behavioral approaches to conservation in the wild (pp. 95-131). Cambridge: Cambridge University Press. 